\title{
Definiciones de los Reparos, Marcas Óseas o Accidentes de la Superficie del Hueso en Anatomía Humana
}

\author{
Definitions of the Landmarks, Bony Marks or \\ Accidentes of the Bone Surface in Human Anatomy
}

\author{
Nicolás Vidal-Seguel'; Ricardo Esteban Miranda Krause²; Marcela Pumeyrau-Solar ${ }^{3}$ \& Bélgica Vásquez
}

VIDAL-SEGUEL, N.; MIRANDA, K. R. E.; PUMEYRAU-SOLAR, M. \& VÁSQUEZ, B. Definiciones de los reparos, marcas óseas o accidentes de la superficie del hueso en Anatomía Humana. Int. J. Morphol., 39(1):335-340, 2021.

RESUMEN: El aprendizaje de la Anatomía puede resultar un proceso dificultoso y poco motivador para el estudiante, esto debido a su complejo vocabulario. Uno de los desafíos iniciales en el proceso de enseñanza-aprendizaje de la Anatomía Humana es el abordaje de los términos generales del sistema musculoesquelético agrupados con los nombres de reparos, marcas óseas o accidentes de la superficie del hueso. Estos suelen ser mencionados con una escasa o nula definición en los recursos bibliográficos. El objetivo de este trabajo fue revisar los recursos bibliográficos que efectivamente cuentan con definiciones de los términos denominados como reparos, marcas óseas o accidentes de la superficie del hueso, con la finalidad de aportan información aclaratoria sobre el uso de estos términos óseos y así facilitar los procesos de enseñanza-aprendizaje de la Anatomía Humana. Los términos utilizados comúnmente para describir reparos, marcas óseas o accidentes de la superficie del hueso fueron analizados en Terminologia Anatomica (FIPAT, 2019) y en cinco textos de Anatomía Humana. Para facilitar la comprensión, los resultados fueron clasificados y tabulados Se pudo evidenciar una escasez de recursos bibliográficos con definiciones de los reparos, marcas óseas o accidentes de la superficie del hueso, la mayoría de estos términos óseos se encontraron en el sistema musculoesquélico, sin embargo, no son exclusivos de este sistema, además, se observaron errores conceptuales en las definiciones de algunos de los términos óseos. Una manera de comprender mejor estos términos, es revisar de manera frecuente la Terminologia Anatomica.

PALABRAS CLAVE: Reparos óseos; Marcas óseas; Accidentes anatómicos; Terminologia Anatomica.

\section{INTRODUCCIÓN}

La enseñanza de la Anatomía Humana tiene como objetivo familiarizar a los estudiantes con un lenguaje técnico-médico necesario para su formación profesional. Los términos anatómicos están contenidos en la Terminologia Anatomica, que es la encargada de unificar todos los términos anatómicos empleados para nombrar, identificar, describir y ubicar espacialmente a los órganos corporales, así como las relaciones entre ellos (Aziz et al., 2002; Díaz Rojo, 2001). No obstante, el aprendizaje de la anatomía humana por parte de los estudiantes puede ser compleja debido a su amplio vocabulario (Guiraldes et al., 2001).

Los estudiantes perciben la enseñanza de la anatomía como una asignatura que contiene una gran cantidad de términos, el que muchas veces no es presentado de una for- ma que propicie una comprensión de los significados reales; situación que puede tributar en un proceso de aprendizaje poco motivador (Torres, 2013). Lo anterior, es un antecedente relevante ya que el factor motivacional posee un gran impacto en los procesos de aprendizaje (Monroy \& Hernández Pina, 2014), siendo un determinante directo en el rendimiento y los logros académicos (Maurer et al., 2013).

Si bien en todas las carreras de las ciencias médicas se enseña Anatomía Humana, esta enseñanza no se imparte de la misma manera, los contenidos son diferentes y la terminología anatómica utilizada difiere considerablemente entre los académicos de diferentes países, entre universidades de un mismo país e incluso entre profesores de la misma universidad o instituto de educación superior.

\footnotetext{
${ }^{1}$ Escuela de Enfermería, Facultad de Salud, Universidad Santo Tomás, Chile.

2 Departamento de Medicina, Facultad de Medicina, Universidad de Atacama, Copiapó, Chile.

${ }^{3}$ Programa de Magíster en Lingüística Aplicada, Facultad de Humanidades y Arte, Universidad de Concepción, Concepción, Chile.

${ }^{4}$ Universidad de Tarapacá, Arica, Chile.
} 
Se han realizado numerosos esfuerzos por facilitar la comprensión y la enseñanza de la anatomía. En la Nomina Anatomica de Basilea (1895), Nomina Anatomica de Jena (1935) y Nomina Anatomica de París (1955) se puede observar el esfuerzo histórico realizado por anatomistas pertenecientes a las sociedades científicas del área morfológica. Este trabajo realizado durante más de 125 años ha tenido como principal objetivo implementar un lenguaje común que permita una real comunicación entre los morfólogos. En las últimas décadas, la $1^{\mathrm{a}}$ edición de la Terminologia Anatomica (FCAT, 1988) y la $2^{\mathrm{a}}$ edición de la Terminologia Anatomica (FIPAT, 2019), han modificado, corregido, retirado y principalmente incorporado nuevos términos.

Dentro de este lenguaje anatómico que parece ser complejo para el estudiante, se encuentran los términos denominados comúnmente como: reparos, marcas óseas o accidentes de la superficie del hueso, definidos en un conjunto según Moore et al. (2017) como marcas donde se insertan tendones, ligamentos y fascias, o registros generados por el paso de elementos neurovasculares. La asimilación por parte de los estudiantes de estos términos corresponde a uno de los desafíos iniciales en el proceso de enseñanza-aprendizaje de la Anatomía, ya que se usan como términos o conceptos generales del sistema musculoesquelético, uno de los primeros en ser tratados en los diversos programas de Anatomía Humana.

Si bien estos términos anatómicos suelen mencionarse en los libros clásicos de Anatomía Humana, en muchos de ellos no se realiza una definición minuciosa, los que son presentados además como términos exclusivos para el sistema musculoesquelético.

En base a lo expuesto, el objetivo de este estudio fue revisar los recursos bibliográficos que efectivamente cuentan con definiciones de los términos denominados como reparos, marcas óseas o accidentes de la superficie del hueso, con la finalidad de aportan información aclaratoria sobre el uso de estos términos óseos y así facilitar los procesos de enseñanza-aprendizaje de la Anatomía Humana.

\section{MATERIAL Y MÉTODO}

Los términos utilizados comúnmente para describir reparos, marcas óseas o accidentes de la superficie del hueso fueron analizados en Terminologia Anatomica (FIPAT) y en cinco textos de Anatomía Humana: Testut \& Latarjet (1969), Chatain \& Bustamante (1986), Pró (2014), Moore et al. y Latarjet \& Ruiz-Liard (2019).
Las definiciones de estos términos óseos que fueron mayormente desarrollados por los textos analizados fueron ordenados y tabulados. Posteriormente, los términos usados para describir reparos, marcas óseas o accidentes de la superficie del hueso fueron clasificados según Testut \& Latarjet.

Para facilitar la comprensión, los términos fueron colocados, cuando fue posible, de acuerdo a Terminologia Anatomica (FIPAT), a excepción de las Tablas.

\section{RESULTADOS}

El análisis de la Terminologia Anatomica y de los libros clásicos de Anatomía constató la existencia de numerosos términos anatómicos que están incluidos como: reparos, marcas, relieves o accidentes en la superficie del hueso, casi todos ellos se encuentran presentes en el sistema muscoloesquelético, sin embargo, muchos de ellos también son utilizados en otros sistemas de cuerpo humano e incluye a glándulas endocrinas, órganos de los sentidos, e integumento común.

En la Tabla I se observan las definiciones para términos óseos señalados como reparos, marcas óseas o accidentes de la superficie del hueso realizadas en tres libros de Anatomía Humana.

El Tratado de Anatomía Humana de Testut \& Latarjet clasifica estos términos en tres grandes pilares: 1) Eminencias o apófisis, 2) Cavidades de los huesos y 3) Agujeros o conductos de los huesos (Tabla II). Sin embargo, no se encuentra una definición de cada uno de estos términos. Por el contrario, los autores declaran que las denominaciones que han recibido las eminencias no articulares, son lo suficientemente expresivas de por sí para hacer innecesaria toda definición.

En el libro de Latarjet \& Ruiz-Liard se pueden encontrar más o menos definidos los reparos óseos. Según estos autores, algunos huesos de la cara y del cráneo presentan cavidades rellenas de aire. Si tienen dimensiones reducidas se les designa celdas, cuando son de un tamaño mayor son senos. Estos mismos autores señalaron que en la superficie de los huesos existen irregularidades, como salientes, entrantes y superficies ásperas. Entre las eminencias extraarticulares señalan que son muy variables, irregulares y rugosas, por lo general, destinadas a inserciones musculares o ligamentosas; su desarrollo varía según la potencia que ejerce el músculo que se inserta en ellas. Estos autores, terminan señalando que a estas irregularidades se las denomina procesos (apófisis), protuberancias, tuberosidades, espinas, crestas y líneas. 
Tabla I. Definiciones para términos incluidos como reparos, marcas óseas o accidentes de la superficie del hueso incluidas en libros de Anatomía Humana de Chatain \& Bustamante, Pró y Moore et al.

\begin{tabular}{|c|c|c|c|}
\hline Término & Chatain \& Bustamante, 1986 & Pró, 2014 & Moore et al., 2017 \\
\hline Cabeza & Segmento de esfera. & Extremo abultado articular. & Extremo articular grande y redondeado. \\
\hline Canal & Túnel. & Depresión lineal. & - \\
\hline Cara, carilla o fosita & - & - & $\begin{array}{l}\text { Área plana y lisa, habitualmente cubierta de } \\
\text { cartílago, donde un hueso se articula con otro. }\end{array}$ \\
\hline Capítulo & - & $\begin{array}{l}\text { Extremo abultado articular como una cabeza, } \\
\text { pero de menor tamaño. }\end{array}$ & Pequeña cabeza articular redondeada. \\
\hline Cóndilo & Superficie articular redondeada. & Saliente articular en un complejo articular & $\begin{array}{l}\text { Área articular redondeada, semejante a un } \\
\text { nudillo; con frecuencia es una estructura par. }\end{array}$ \\
\hline Conducto & $\begin{array}{l}\text { No pertenece al sistema óseo y es una } \\
\text { estructura tubular que contiene una sustancia } \\
\text { líauida. }\end{array}$ & Depresión lineal & - \\
\hline Conductillo & - & - & - \\
\hline Cresta & Borde prominente y agudo. & Reborde óseo ala rgado & Reborde óseo. \\
\hline Incisura & 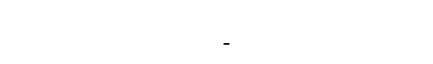 & Muesca entrante en un borde. & Muesca en el borde de un hueso. \\
\hline Epicóndilo & $\begin{array}{l}\text { Prominencia pequeña que está situada en la } \\
\text { proximidad de un cóndilo. }\end{array}$ & $\begin{array}{l}\text { Relieve óseo rela cionado por proximidad con } \\
\text { un cóndilo. }\end{array}$ & Eminencia superior a un cóndilo. \\
\hline Espina & Elevación o proyección puntiaguda. & Protrusión puntiaguda. & Proceso semejante a una espina. \\
\hline Hámulo & Proyección en forma de gancho. & - & - \\
\hline Fóvea & Área articular pequeña. & - & - \\
\hline Foramen & Orific io o apertura. & Aberturas por donde pasan los vasos. & Paso a través de un hueso. \\
\hline Fosa & Depresión más o menos profunda. & - & Hueco o área deprimida. \\
\hline Línea & Borde prominente y agudo, pero bajo. & Una protrusión ósea lineal. & Elevación lineal. \\
\hline Maléolo & - & - & Proceso (apófisis) redondeado. \\
\hline Proceso & Elevación o proyección grande y redondeada. & $\begin{array}{l}\text { (apófisis) protrusión alargada, puede ser de } \\
\text { inserción. }\end{array}$ & $\begin{array}{l}\text { Extensión o proyección con un propósito } \\
\text { principal, forma característica o que se } \\
\text { extiende hacia una dirección particular. }\end{array}$ \\
\hline Protuberancia & - & Prominencia más o menos redondeada. & Abultamiento o proyección ósea. \\
\hline Surco & Depresión alargada. & Depresión lineal. & Depresión alargada. \\
\hline Trocánter & - & - & Gran elevación roma. \\
\hline Tróclea & Forma de polea. & - & $\begin{array}{l}\text { Proceso articular semejante a un carrete que } \\
\text { actúa como una polea. }\end{array}$ \\
\hline Tubérculo & Elevación o proyección grande y redondeada. & Protuberancia ósea de menor tamaño. & Pequeña eminencia elevada. \\
\hline Tuberosidad & Elevación o proyección grande y redondeada. & $\begin{array}{l}\text { Protuberancia ósea más bien redondeada con } \\
\text { un relieve rugoso. }\end{array}$ & Gran elevación redondeada. \\
\hline Apófisis & - & $\begin{array}{l}\text { Protrusión desarrollada en una epífisis, con un } \\
\text { centro de osificación propio. }\end{array}$ & - \\
\hline
\end{tabular}

Tabla II. Clasificación de términos incluidos como reparos, marcas óseas o accidentes de la superficie del hueso según Testut \& Latarjet.

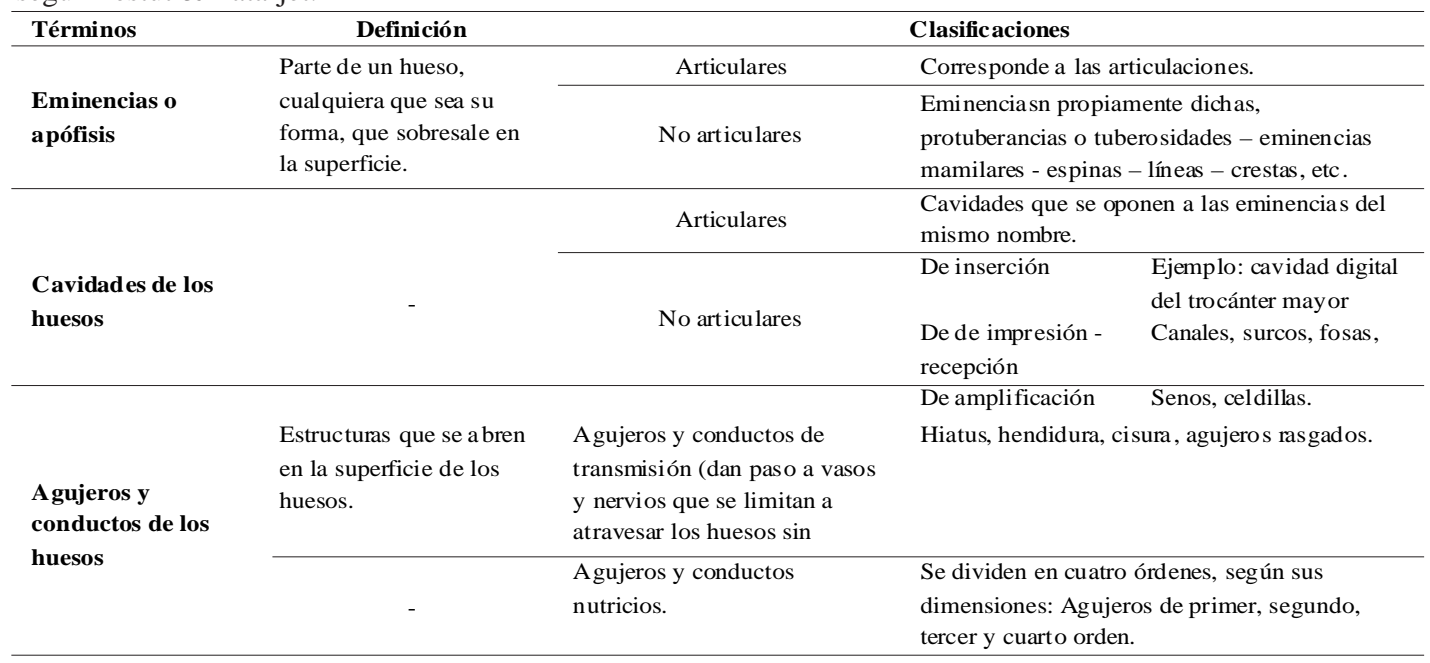




\section{DISCUSIÓN}

Los reparos, marcas óseas o accidentes de la superficie del hueso son de amplio uso en anatomía humana y como es de esperar casi todos ellos se encontraron presentes en el sistema musculoesquelético (huesos, articulaciones y sistema muscular), sin embargo, ellos no son exclusivos para este sistema, sino que, por el contrario, la utilización de estos términos transciende a todos los sistemas del cuerpo humano.

Según los lingüistas, los términos científicos se diferencian de los vocablos del lenguaje común por su precisión, es decir, por tener claramente delimitados sus significados (López \& Terrada, 1990). Al respecto, la anatomía como rama de las Ciencias Morfológicas, ha tratado de unificar criterios al momento de proponer nuevas estructuras o renombrar términos existentes en la Terminologia Anatomica (Roa et al., 2016). En base a esto, se sugiere que los términos anatómicos cumplan con un lenguaje descriptivo, específico, universal, inequívoco y preciso (Montemayor et al., 2016), delimitando los significados de cada término y de esta forma favorecer entre otros aspectos, el proceso de enseñanza-aprendizaje. Sin embargo, se puede constatar que un gran número de textos de acceso común para los estudiantes no cuentan con definiciones anatómicas para los llamados reparos, marcas óseas o accidentes de la superficie del hueso.

Una clara y exacta definición puede contribuir a evitar lo señalado por Monrroy et al., quienes indicaron que el estudiante podría centrase solo en la memorización de los términos al no encontrar conexiones entre ideas o lenguaje previo, generando de esta forma un aprendizaje superficial.

Es necesario destacar que textos en otros idiomas cuentan con definiciones de estos reparos óseos. Por ejemplo, en el libro de Anatomía Humana de van de Graaff (2003) en la $6^{\mathrm{a}}$ edición en inglés, como en la $1^{\mathrm{a}}$ edición en portugués, presenta una tabla con las características de la superficie del hueso. Las separa en tres categorías: $1^{\circ}$ superficies articulares (cóndilo, fóvea, cabeza); $2^{\circ}$ depresiones y aberturas (alvéolo, fisura, foramen, fosa, seno, surco) y $3^{\circ}$ prominencias no articulares (cresta, epicóndilo, proceso, rama, espina, trocánter, tubérculo y tuberosidad). Otro ejemplo, es el libro de Anatomía Clínica de Snell (1999) quien define una serie de "marcas en la superficie de los huesos", las divide en: $1^{\circ}$ Elevación linear (línea y cresta); $2^{\circ}$ Elevación arredondeada (tubérculo, protuberancia, tuberosidad, maléolo y trocánter); $3^{\circ}$ Elevación aguda (espina o proceso espinoso, proceso estiloide); $4^{\circ}$ Extremidades expandidas para articulación (cabeza, cóndilo, epicóndilo [una promi- nencia situada luego encima de un cóndilo]); $5^{\circ}$ Pequeña área plana para articulación (faceta); $6^{\circ}$ Depresiones (incisura, surco, fosa); $7^{\circ}$ Aberturas (fisura, foramen, canal y meato). Nos parece muy interesante este tipo de agrupamiento de las marcas de la superficie de los huesos, pero también llama la atención la dualidad del término proceso, al cual le agrega estiloide. En el libro, el término proceso es utilizado en muchas estructuras anatómicas y no solamente para los procesos estiloide del radio, la ulna o del hueso temporal.

Los resultados expuestos en este trabajo aportan información aclaratoria sobre el uso de los términos incluidos como reparos, marcas óseas o accidentes de la superficie del hueso, sin embargo, bajo la definición generada por Testut \& Latarjet existen ciertos términos que podrían generar confusión, como son los casos: 1) eminencia y proceso, 2) protuberancia y tuberosidad y 3) cavidad y fosa. Estas parejas de términos son tomadas como sinónimos por dichos autores. Si bien las definiciones pueden ser similares, su uso en anatomía es distinto.

Cabe destacar que los reparos, marcas óseas o accidentes de la superficie del hueso cumplen la función de adjetivar, acompañando a una estructura (sustantivo). En la Terminologia Anatomica (FIPAT) se encuentran todos estos términos.

Uno de los términos que más llama la atención en Terminologia Anatomica (FIPAT) es el de apófisis (término 397), y que para muchos autores clásicos correspondería a un proceso. Incluso en todas las figuras anatómicas del libro de Pró se señala a estos accidentes óseos como apófisis y entre paréntesis proceso. Paradojalmente, en la Terminologia Anatomica (FIPAT) no se encuentra el término apófisis acompañando a una determinada estructura, solo se encuentra formando parte de los sinónimos latinos cigoapófisis superior y cigoapófisis inferior, que corresponden a los procesos articulares superiores e inferiores de las vértebras, respectivamente. Estos términos de cigoapófisis superior e inferior se encuentran mencionados en algunos textos como el de Anatomía de Gray (Williams et al., 1995) y corresponden a las proyecciones a partir del arco vertebral en las uniones pedículolaminares. Según la definición de Pró el término apófisis resulta controversial, ya que la define como una protrusión desarrollada en una epífisis, pudiendo ser de inserción.

El término proceso, del inglés, es un término masculino, el cual de acuerdo al Diccionario de Términos Médicos de la Real Academia Nacional de Medicina (2011), 
significa protuberancia y se encuentra documentado en español desde 1246 en significados no anatómicos; fue reintroducido y documentado en francés en sentido anatómico desde 1560. Apófisis sería su sinónimo, sin embargo, una observación de acepción señala que puede suscitar rechazo por considerarse anglolatinismo ajeno a la nomenclatura española tradicional.

$\mathrm{Al}$ revisar la literatura anatómica en inglés y otros idiomas, sean estos libros o artículos, llama poderamente la atención que la traducción al idioma español estén tan mal realizada y no siguiendo la Terminologia Anatomica (FIPAT), lo que hace aún más difícil la comunicación científica. El término proceso es traducido al español en la mayoría de los casos como apófisis; el término margen es traducido como borde, y así muchos otros términos de innumerables estructuras anatómicas son mal empleados e incorrectamente traducidos al español.

Una manera de comprender mejor los diferentes términos incluidos como reparos, marcas óseas o accidentes de la superficie del hueso, sería revisar de manera frecuente la Terminologia Anatomica (FIPAT), estudiar dichos términos y realizar un análisis crítico de ellos para poder dar una explicación más clara a los estudiantes del área de las Ciencias de la Salud. Como lo señalaron Chmielewski \& Domagala (2020) es importante recordar que la terminología anatómica es parte de la terminología científica que merece la debida consideración y puede actualizarse y mejorarse cuando sea necesario. Sin embargo, como lo señalaron Wen et al. (2019) la actual Terminologia Anatomica (FIPAT), se encuentra redactada en latín y a partir de ella se realiza la traducción al idioma correspondiente, entonces, al igual que estos autores, cabe preguntarse si la traducción al español es las más adecuada y correcta para denominar esas estructuras y reparos, marcas o accidentes en las superficies de los huesos que aparecen publicados en los textos de anatomía disponibles en Chile.

A pesar de la definición que realizan algunos autores sobre los diferentes términos anatómicos incluidos como reparos, marcas óseas o accidentes de la superficie del hueso existen muchos errores conceptuales, por ejemplo, el término cavidad glenoidea que no es el correcto y que de acuerdo a Terminologia Anatomica (FIPAT), debería ser fosa glenoidea.

Finalmente, destacamos la importancia de la comprensión de las etimologías latinas y griegas (Vidal et al., 2019) en el análisis de los reparos, marcas o accidentes en las superficies de los huesos, ya que este enfoque permitirá definir de mejor manera estos términos óseos y en consecuencia mejorar la enseñanza-aprendizaje de la Anatomía Humana.
VIDAL-SEGUEL, N.; MIRANDA, K. R. E.; PUMEYRAU-SOLAR, M. \& VÁSQUEZ, B. Definitions of the landmarks, bony marks or accidents of the bone surface in human anatomy. Int. J. Morphol., 39(1):335-340, 2021.

SUMMARY: Learning Anatomy can be a difficult and unmotivating process for the student, due to its complex vocabulary. One of the initial challenges in the teaching-learning process of Human Anatomy is the approach to the general terms of the musculoskeletal system grouped with the names of repairs, bone marks or accidents on the surface of the bone. These are usually mentioned with little or no definition in bibliographic resources. The objective of this work was to review the bibliographic resources that effectively have definitions of the terms known as repairs, bone marks or accidents on the bone surface, in order to provide clarifying information on the use of these bone terms and thus facilitate the teaching-learning processes of Human Anatomy. The terms commonly used to describe repairs, bone marks or accidents on the bone surface were analyzed in Terminologia Anatomica (FIPAT, 2019) and in five texts on Human Anatomy. To facilitate understanding, the results were classified and tabulated. There was a lack of bibliographic resources with definitions of the repairs, bone marks or accidents on the bone surface. Most of these bone terms were found in the musculoskeletal system. However, they are not exclusive to this system, and conceptual errors were observed in the definitions of some of the bone terms. One way to better understand these terms is to frequently review the Terminologia Anatomica.

KEY WORDS: Bone repairs; Bone marks; Anatomical accidents; Terminologia Anatomica.

\section{REFERENCIAS BIBLIOGRÁFICAS}

Aziz, M. A.; McKenzie, J. C.; Wilson, J. S.; Cowie, R. J.; Ayeni, S. A. \& Dunn, B. K. The human cadaver in the age of biomedical informatics. Anat. Rec., 269(1):20-32, 2002.

Chatain, L. I. \& Bustamante, B. J. Anatomía Macroscópica Funcional y Clínica. México D. F., Addison-Wesley Iberoamericana, 1986.

Chmielewski, P. P. \& Domagala, Z. A. Terminologia Anatomica and its practical usage: pitfalls and how to avoid them. Folia Morphol., 79(2):198-204, 2020.

Comité International de la Nomenclature Anatomique. Nomina Anatomica. París, 1955.

Díaz Rojo, J.A. La terminología médica: diversidad, norma y uso. Med. Interam., 2(4):40-6, 2001.

Federative Committee on Anatomical Terminology (FCAT). Terminologia Anatomica: International Anatomical Terminology. Stuttgart, Georg Thieme Verlag, 1998.

Federative International Programme for Anatomical Terminology (FIPAT). Terminologia Anatomica. 2nd ed. FIPAT.library.dal.ca. Federative International Programme for Anatomical Terminology, 2019.

Guiraldes, H.; Oddó, H.; Mena, B.; Velasco, N. \& Paulos, J. Enseñanza de la anatomía humana: experiencias y desafíos en una escuela de medicina. Rev. Chil. Anat., 19(2):205-12, 2001.

Latarjet, M. \& Ruiz-Liard. A. Anatomía humana. $5^{\text {a }}$ ed. T. 1. Buenos Aires, 2019.

López, P. J. M. \& Terrada, F. M. L. Introducción a la terminología médica. Barcelona, Salvat, 1990. 
Maurer, T.W.; Allen, D.; Gatch, D.B; Shankar, P.; Sturges, D. Students' academic motivations in three disciplines. J. Scholarship Teach Learn., 13(5):77-89, 2013.

Monroy, F. \& Hernández Pina, F. Factores que influyen en los enfoques de aprendizaje universitario. Una revisión sistemática. Educación XX1., 17(2):105-24, 2014

Montemayor, B.; Herrera, I. \& Soto, A. Análisis del uso de la Terminología Anatómica entre los Estudiantes de la Asignatura Anatomía de la Licenciatura en Medicina, de la Facultad de Medicina de la Universidad Nacional Autónoma de México. Int. J. Morphol., 34(4):1280-4, 2016.

Moore, K. L.; Dalley, A. F. \& Agur, A. M. R. Anatomía con Orientación Clínica. 8a ed., Philadelphia, Wolters Kluwer, 2017.

Pró, E. A. Anatomía Clínica. $2^{\mathrm{a}}$ ed. Buenos Aires, Médica Panamericana, 2014.

Real Academia Nacional de Medicina. Diccionario de Términos Médicos. Panamericana, Madrid. 2011.

Roa, I.; Vásquez, B. \& Contreras, M. Eponyms persistence in Terminologia Histologica. Int. J. Morphol., 34(4):1245-52, 2016.

Snell, R. S. Anatomia Clínica para estudantes de medicina. Guanabara Koogan, Rio de Janeiro, 1999.

Testut, L. \& Latarjet A. Anatomía Humana. T. 1. Barcelona, Salvat Editores, 1969.

Torres, M. N. Y. Enseñanza de Anatomía: Una Experiencia a Partir de Cuestionamientos Propuestos en Situaciones Contextuales. Escenarios. 11(1):131-8, 2013.

van de Graaff, K. M. Anatomia Humana. $1^{\text {a }}$ ed. Manole, São Paulo, 2003.

Vidal-Seguel, N.; Pumeyrau-Solar, M.; Lizama-Pérez, R.; Muñoz-Cofré, R.; Conei, D.; Nicholson, C. \& del Sol, M. Análisis del Lexema Neur(o) y sus derivados en el aprendizaje de la anatomía. Int. J. Morphol., 37(4):1517-21, 2019.

Wen, S.; del Sol, M. \& Nicholson, C. Mylos root in Terminologia Anatomica. Int. J. Morphol., 38(1):126-128, 2020.

Williams, P. L.; Warwick, R.; Dyson, M. \& Bannister, L. H. Gray Anatomia. $37^{\mathrm{a}}$ ed. Guanabara Koogan, Rio de Janeiro, 1995.
Dirección para correspondencia:

Dra. Bélgica Vásquez

Universidad de Tarapacá

Arica

CHILE

Email: bvasquezp@uta.cl

Dirección para correspondencia:

Nicolás Enrique Vidal Seguel

Universidad Santo Tomás

Sede Temuco

CHILE

Email: nvidal4@santotomas.cl

Recibido : 13-10-2020

Aceptado: 09-11-2020 